\title{
RESEARCH ON COVID-19 FROM BIOPHYSICAL PERSPECTIVE
}

\author{
Tika Ram Lamichhane ${ }^{1 *}$, Madhav Prasad Ghimire ${ }^{1,2 *}$ \\ ${ }^{I}$ Central Department of Physics, Tribhuvan University, Kathmandu, Nepal \\ ${ }^{2}$ Condensed Matter Physics Research Center, Butwal-11, Rupandehi, Lumbini, Nepal \\ *Corresponding authors: tika.lamichhane@cdp.tu.edu.np
}

\begin{abstract}
In this report, we discuss the current aspect of biophysics which is an essential field to investigate the structural and related properties of SARS-CoV-2 components, possible drugs likeliness and their interactions, and mathematical as well as clinical data modeling. We have emphasized that a mathematical model generates the sigmoid logistic equation that predicts the total coronavirus cases and deaths in a model country with population averaged from the most vulnerable countries, such as India or USA, where the COVID-19 cases are still spiking up and from the countries which are on the process of recovering from the current pandemic. Further, the current status of COVID-19 research has been explained to understand its impacts taking Nepal as the reference country having low-income. In particular, focus is on biophysical study on structural analysis of novel coronavirus main protease which is found to influence the radial distributions of H-bonding atom pairs when interacting with the inhibitors N3. Explorations of the variations of physical parameters are found to be important in drug selective mechanism against COVID-19 infections.
\end{abstract}

Keywords: coronavirus - structural analysis - mathematical and clinical data modeling - drugs - viral proteins

\section{INTRODUCTION}

In recent days, livelihood of the people around the globe is under threat due to a severely increasing rate of an infectious disease called novel coronavirus disease (COVID-19). Few types of coronavirus diseases named as Severe Acute Respiratory Syndrome (SARS), Middle East Respiratory Syndrome (MERS) and COVID-19 cause mild to severe respiratory tract infections in mammals and birds. As coronavirus has single stranded RNA 
genome, it is categorized as an RNA virus. Human being suffering from COVID-19 is found to have cough, fever, shortness of breath and tiredness as the most common symptoms (Jin, et al., 2020). The infection by novel coronavirus $(\mathrm{nCoV})$ or SARS-CoV-2 starts when its spike glycoprotein gets bind to the host cell receptor. By endocytosis, the coronavirus enters into the cell cytoplasm. The genome organization for a coronavirus is 5'-untranslated region (UTR)-spike (S)-envelope (E)-membrane (M)nucleocapsid (N)-3'-UTR poly tail. The components of SARS-CoV-2 are shown in Figure 1. The reading frames encode four types of proteins: S, E, $\mathrm{M}$ and $\mathrm{N}$. The viral genome acts as mRNA which is directly translated by the host cell's ribosomes into the overlapping polyproteins ppla and pplab (Fehr, et al., 2015; Mousavizadeh, et al., 2020).

As of June 22, 2020, there are at least 8,860,331 confirmed cases and 465,740 confirmed deaths due to COVID-19 pandemic around the world (World Health Organization, 2020). In the context of Nepal, there are 9,561 total cases and 23 total deaths due to COVID-19 till date (Worldometers, 2020); Ministry of Health and Population, 2020). Confirmed COVID-19 cases show an increasing trend in South East Asia region with India and Bangladesh reporting the highest number of cases. The daily cases are raising, however fatality of this disease seems to be lower in Nepal $\left(66^{\text {th }}\right.$ country in the world by COVID-19 pandemic) than other countries like India, USA, Brazil, UK, France, Mexico, Italy, Spain and Belgium as reported as of $23^{\text {rd }}$ June, 2020.

The nCoV carriers can transmit COVID-19 to others through fecal-oral route when they come closer to each other (Chan et al., 2020; Li, et al., 2020). The degree of infection depends on the interaction of viral S protein with its complementary cell receptor-angiotensin-converting enzyme 2 (ACE2) in the respiratory tract. The elder people who are immunocompromised are at higher risk. The protective measures according to WHO guidelines are to maintain social distancing, wash hands frequently or use hand sanitizer, cover face with mask, use PPE for healthcare workers and apply strict hygiene measures in hospitals (Cascella, et al., 2020). 


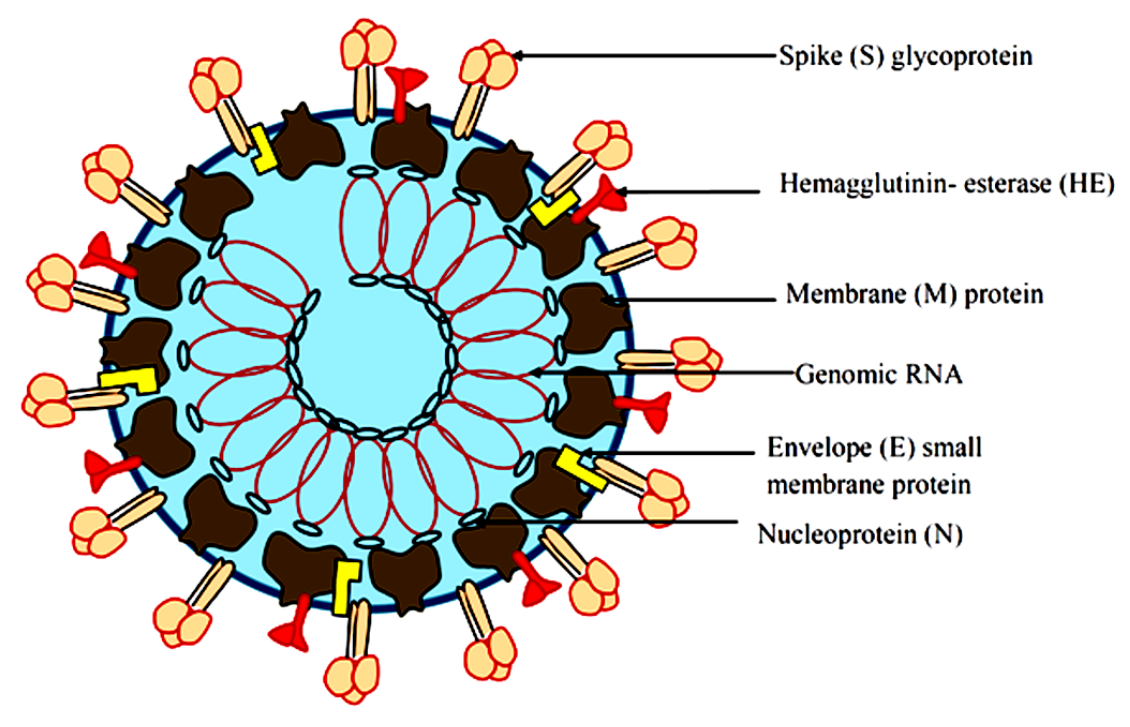

Figure1: Schematic structure of SARS-CoV-2 as coronal appearance consisting of enveloped visions having diameter $\sim 120 \mathrm{~nm}$

\section{COMPUTATIONAL AND CLINICAL DATA MODELING}

Extensive research on $\mathrm{nCoV}$ is ongoing to resolve the current pandemic by using the epidemic analysis and its transmissibility. In modeling, data is the first step to develop any diagnostic tool or treatment. The dataset and its analysis help in understanding the dynamics of the disease based on which one can prepare for better treatments. Way back in 1920 a simple mathematical model named SIR (Susceptible-InfectiousRecovered) was first used which consider a set of equations to simulate the disease developing over time in human beings. Basing on the same model, numerous models have been developed.

Study suggests that mathematical modeling techniques can be harnessed to develop successful control strategies and can have the potential to probe the intractable complexity of infectious disease dynamics (Heesterbeek, et al., 2015). For characterizing the epidemic COVID-19 outbreak in terms of effectiveness of various measures such as effects of lock-down, quarantine, isolation, transmission risk, predicting the patient number and chances of recovery, an extended version of SIR model called the susceptible exposed infectious recover model (SEIR) has been widely used (Peng, et al., 2020; Vespignani et al., 2020). 
Recently, Cohen et al., (2020) collected image data of initial COVID-19 patients created by assembling the medical images that contains 123 frontal view X-rays. By comparing the public database of pneumonia with the chest X-rays or CT scans of COVID-19, computational analysis was done to study how the radiological findings vary from other types of pneumonia.

To understand the deep learning, machine learning and statistical, mathematical modeling of the clinical data are important in order to forecast the impact of COVID-19 pandemic. Here, we try to briefly update the status of COVID-19 as of $21^{\text {st }}$ June, 2020 to draw a strategic possible situation that can arise in upcoming days.

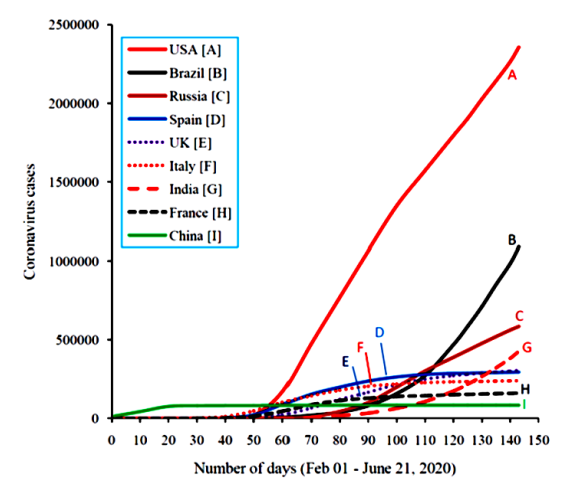

(a)

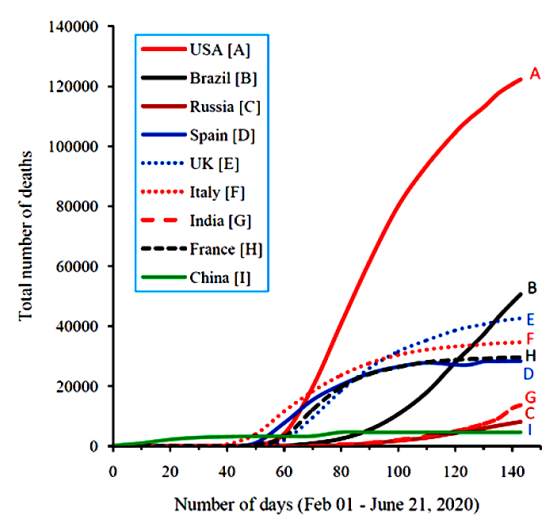

(b)

Figure 2: (a) Total coronavirus cases and (b) total deaths due to COVID-19 in the vulnerable nine countries

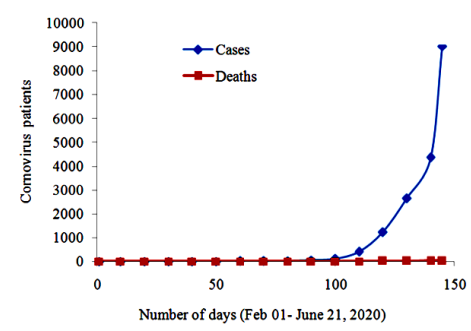

(a)

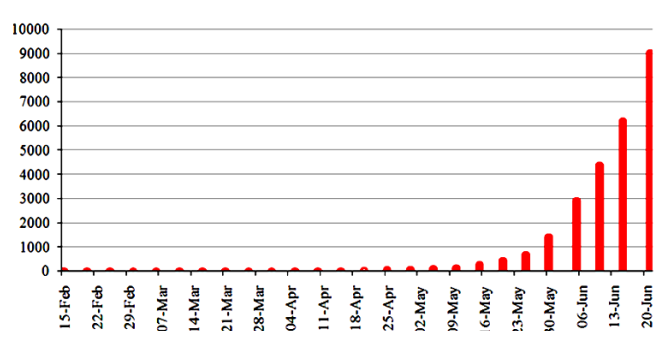

(b)

Figure 3: (a) Weekly reports of total coronavirus cases and (b) comparison between total cases and deaths due to COVID-19 in Nepal (as of June 21, 2020 , total death $=23$ ) 
As of June 21, 2020, the total cases and deaths of COVID-19 in nine vulnerable countries with the spiking cases or are going to be recovered are plotted separately as shown in Figure 2 (Worldometers, 2020). As shown, countries like USA, Brazil, Russia and India has significant rise of the cases. On one hand, when China had a recovery phase after 20 days, countries like Spain, France, UK and Italy have the curves almost flattened after about 60 days from the outbreak. The different nature so observed seems to be particularly dependent on how the pandemic situation is taken care by the respective/concerned governments.

In the context of our country Nepal, according to the Ministry of Health and Population, Nepal, already performed 181,371 PCR and 268,103 RDT tests wherein about 2,148 caseswere recovered, and 23 patients were died due to COVID-19 as of June 22, 2020. The report of the ministry further mention that 90,730 people are in quarantine and 7,390 patients are in isolation. The nature of COVID-19 cases is found to rise exponentially, however the number of deaths is small as plotted in Figure 3. The nCoV cases begin to rise only after 100 days of starting the worldwide outbreak since Feb 1, 2020. Most of the cases are related to the people coming from other countries which are suffering from this pandemic. The community transfer of this disease seems to be low for now in Nepal. The number of cases and fatalities depends on the effectiveness of the disease control management, testing, health awareness of the people and their underlying health conditions.

By taking an average from the COVID-19 cases in nine different countries as mentioned in Figure 2, we have modelled an equation to predict the total cases and the deaths before the recovery from this disease in a virtual country having the mean population of 416,531,900. The best fitted sigmoid function has the equation:

$$
y=\frac{a}{1+e^{-k\left(x-x_{c}\right)}}
$$

where $y=$ total coronavirus cases, $x=$ time from which the pandemic begins, $a=$ amplitude or $y$-value at the upper asymptotic point, $k=$ coefficient of this sigmoid function and $x_{c}=$ central $x$-value at $y=a / 2$. As plotted in Figure 4, in the model country, $a=744,908$ $\pm 48541, x_{c} 111 \pm 4$ days and $k=0.04 \pm 0.003$ representing for the total coronavirus cases. Furthermore, the parameters representing the deaths status in such a country, $a=37073 \pm 1015, x_{c} 90 \pm 2$ days and $k=0.06$ 
\pm 0.004 . For this curve best fitted over the mean cases obtained from the nine countries (see Figure 2), the coefficient of determination is $R^{2}=0.99$. This curve has been modeled by using Origin-2017 (version 94E, OriginLab Corporation, USA).

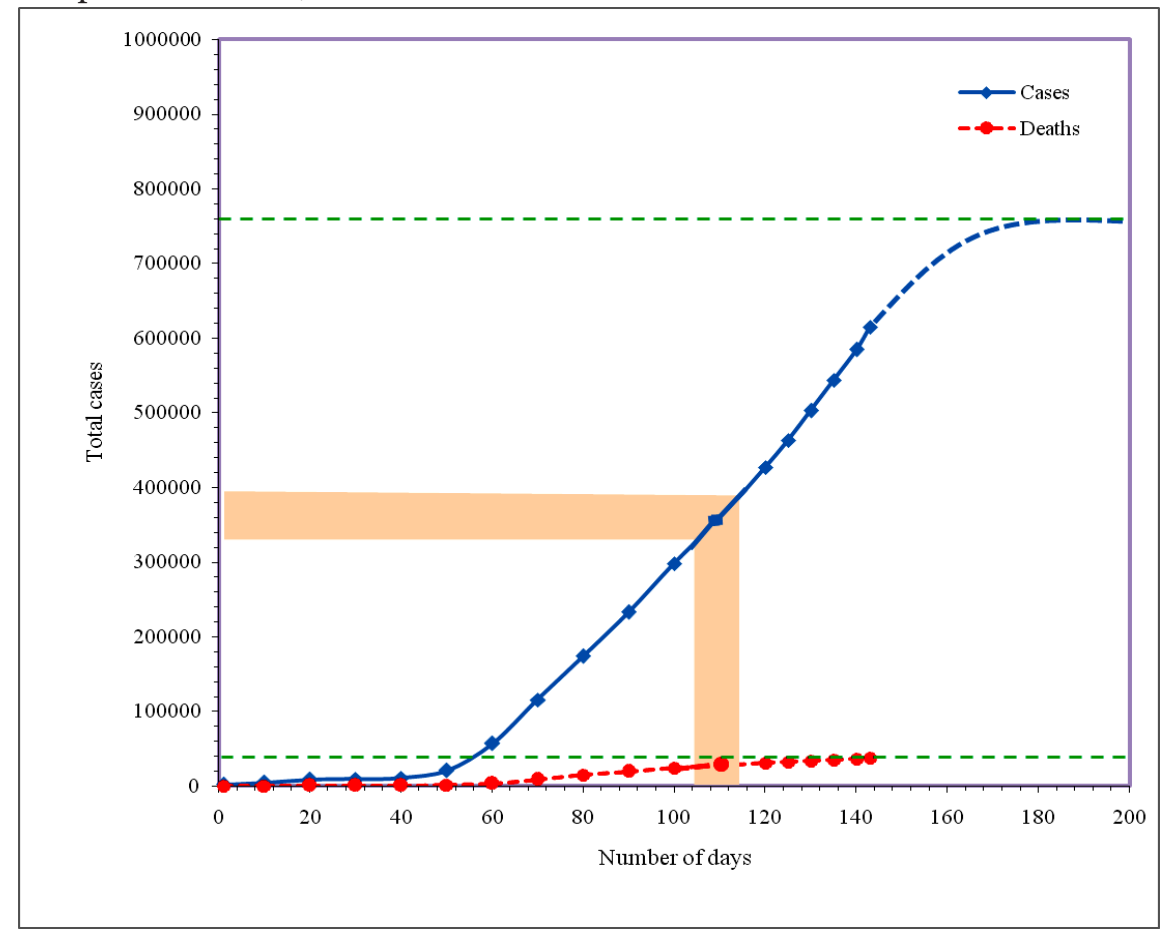

Figure 4: Trend lines of the coronavirus cases and deaths due to COVID-19 in a vulnerable model country having population 416,531,900 averaged from the nine countries mentioned in Figure 2.

\section{SARS-COV-2 STRUCTURAL ANALYSIS}

The interaction between ACE2 and trimeric spike proteins in the receptor binding domain of SARS-CoV-2 is a basis of therapeutic target. The spike protein mediated entry into the cells can be potently inhibited by the polyclonal antibody that is elicited upon vaccination (Walls, et al., 2020). The structural analyses with regard to the genetic sequences, function and antigenicity of SARS-CoV-2 spike (S) glycoprotein in combination with ACE2 have been explained by Walls, et al.(2020) and Yan, et al.(2020). The crystal structure of the receptor-binding domain (RBD) of the spike protein of SARS-CoV-2 in complex with ACE2 can be obtained from the protein data bank (PDB) code: 6LZ.PDB (Shang, et al., 2020) which is as shown 
in Figure 5-b as a cartoon view. The main protease (MP) of SARS-CoV-2 is a key enzyme that has a pivotal role in mediating viral replication and transcription and also acts as a drug target. The structure of MP liganded with inhibitor N3 has been shown in Figure 5-a that is obtained from 6LU7. PDB (Jin, et al., 2020). The structural analysis as well as viral protein-drug interaction can be studied by the methods of computation as explained in the recent literatures (Lamichhane and Ghimire, 2020; Wu, et al., 2020; Muralidharan, et al., 2020; Al-Khafaji , et al., 2020; Mirza, et al., 2020).

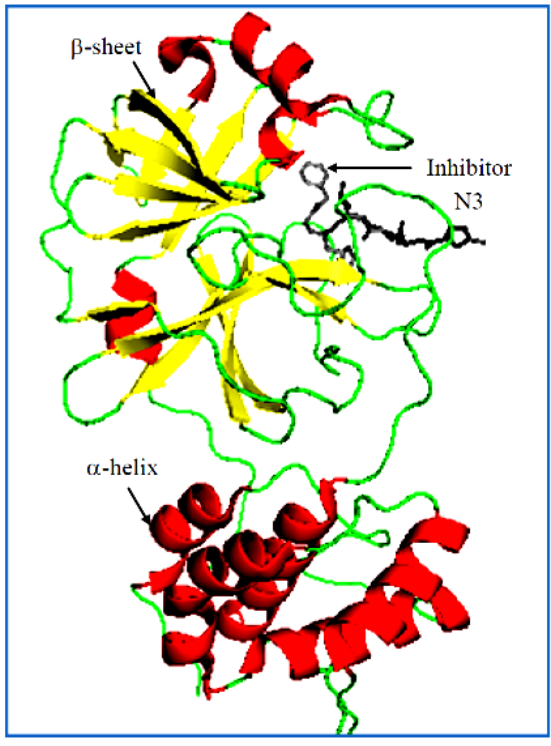

(a)

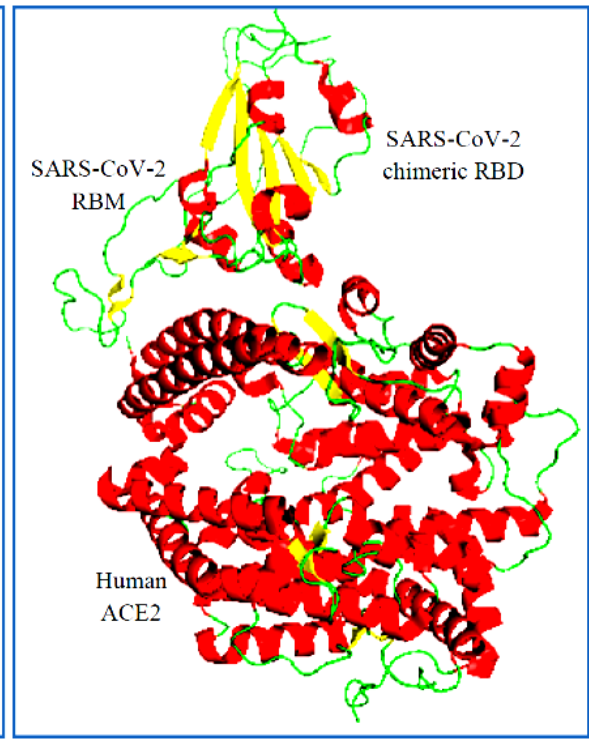

(b)

Figure 5: Structures of (a) nCoV main protease liganded with inhibitor N3 [6LU7.PDB], and (b) nCoV spike protein in complex with ACE2 [6LZG. PDB]

According to Lamichhane and Ghimire (2020), the structural analysis of $\mathrm{nCoV}$ main protease (MP) liganded with inhibitor N3 has been performed in terms of the physical parameters such as root mean square deviation, root mean square fluctuation, radius of gyration, residue velocity, dihedral angle distributions, radial distribution function (RDF) and $\mathrm{H}$-bonding, and the interactions between MP residues in the ligand binding domain (LBD) and inhibitor N3 have been studied in terms of electrostatic and van der Waals energies by means of MD simulations. The conformations of $\mathrm{nCoV}$ related proteins or enzymes are found to change under their interactions with the antiviral drugs or inhibitors which can be studied from 
the biophysical aspects. Exploration of the variations of physical parameters are important in drug selective mechanism against COVID-19 infections. As adapted from Lamichhane and Ghimire (2020), Figure 6 shows how the radial distances between $\mathrm{H}$-bonding atom pairs changes while interacting with the MP-LBD residues with a possible drug like inhibitor N3. Here, the flat RDFs ranging from 1.5 to $6.5 \AA$ are the indicators of breaking and reforming $\mathrm{H}$-bonds during the atomic interactions.

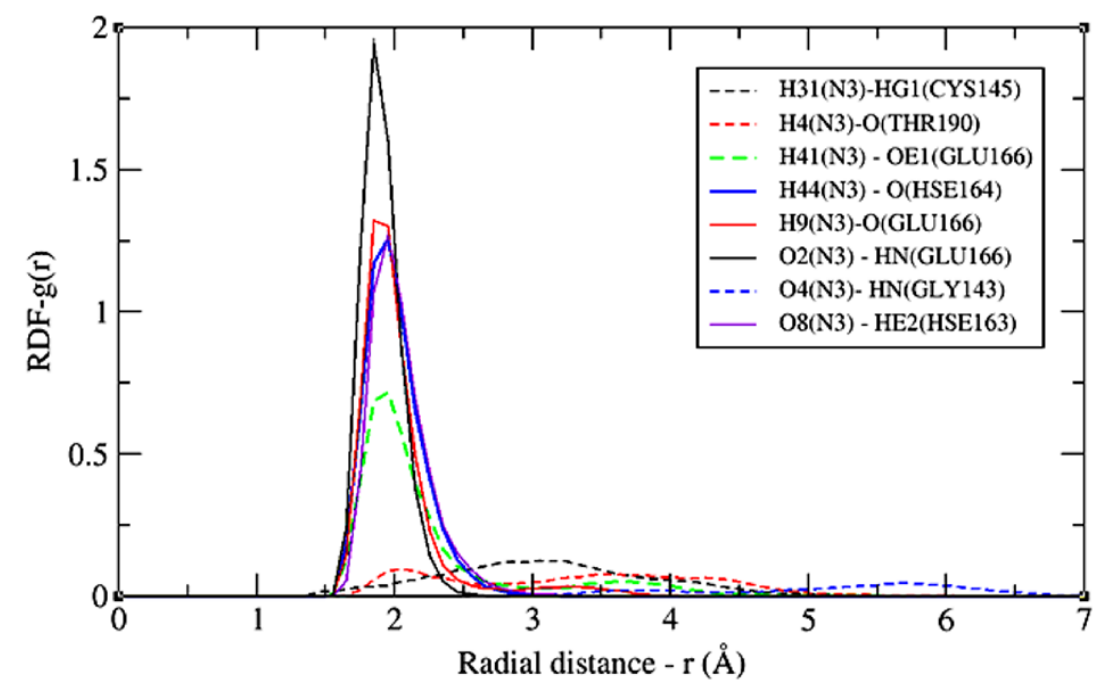

Figure 6: Radial distribution functions of the interacting atom pairs between N3 and MP-LBD residues that make H-bonds to each other except the atom pair: H31(N3)-HG1(CYS145) obtained from the equilibrating MD simulations of MP-N3 complex in the cellular environment (Lamichhane and Ghimire, 2020)

\section{POSSIBLE DRUGS UNDER STUDY}

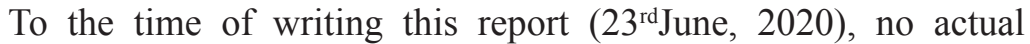
drugs or vaccines has been prescribed so far by WHO for COVID-19 treatment. Several drugs or inhibitors of SARS-CoV-2 such as inhibitor N3 (Jin, et al., 2020; Lamichhane and Ghimire, 2020) remdecivir (Wang, et al., 2020) favipiravir (Cai, et al., 2020), arbidol (Dong, et al., 2020) and dexomethasone (Heidi Ledford, Nature News 2020)are still under preclinical or clinical practices. It is necessary to test the efficacy and safety of the drugs or vaccines before prescribing them for the general public to 
investigate the medicine which actually works against COVID-19. Some steroids like dexamethasone suppress the immune system which can provide relief for the critically ill patients whose lungs are ravaged by an overactive immune response that sometimes manifests in severe cases of COVID-19 (Heidi Ledford, Nature News, 2020). The researchers design the drugs computationally by analyzing the binding properties and their interactions with the viral receptor proteins; they search for the biochemical details, efficacy and safety of such drugs and do trial in other animals such as rat, rabbit, monkey and dogs before the clinical trials in humans.

\section{COVID-19 RESEARCH IN NEPAL}

As of 22 March, 2020, only one coronavirus case had been reported in Nepal (Worldometers, 2020). He is a 32-year-old male student who had returned back from Wuhan, China. He was kept isolated and gave supportive treatment for four days in Sukraraj Tropical and Infectious Disease Hospital (STIDH), Teku, Kathmandu. Since there was no complication except high fever $\left(102^{\circ} \mathrm{F}\right)$, he was discharged by requesting to remain under selfquarantine (Shrestha, et al., 2020). As of 19 April, the coronavirus cases reached 31 without any death. On 24 April, 2020, Nepal government declared countrywide lock-down. However, the cases rise exponentially as shown in Figure 3 reaching 9561 total cases and 23 total deaths due to COVID-19 on 22 June, 2020 (Worldometers, 2020). This scenario reflects the chances of community outbreak in Nepal. Therefore, the preventive measures such as those increasing diagnostic tests, contact tracing, good hospital management and public awareness to maintain social distance, cover with face masks, wash hands or use hand sanitizers and keep selfquarantined are required in order to stop the community outbreak. The people of old age or having underlying health conditions are most vulnerable towards COVID-19 infections. Some research works have been reported from Nepal exploring the public health response (Shrestha, et al., 2020), public health implications (Asim, et al., 2020; Pun, et al., 2020), preventive measures and government preparation (Khadka, et al., 2020; Koirala, et al., 2020), and impact on economy and education (Koirala, et al., 2020; Acharya, et al., 2020). In addition, a research with particular focus on the structural analysis of COVID-19 main protease and its interaction with the inhibitor N3 has been performed by using the biophysical aspects of MD simulations (Lamichhane and Ghimire, 2020). Also, the filtering efficiency and effectiveness of face masks for preventing COVID-19 are explained recently by Neupane et al. $(2019,2020)$. Their report suggest that the 
filtering efficiency of the mask depends on pore size, shape, clearance and number density and its effectiveness depends on breathability, facial fitness and environmental contaminants. The use of PPE is one of the important measures to control the droplet and aerosol mediated transmissions (Somsen, et al., 2020).

\section{CONCLUSIONS}

The worldwide outbreak of the COVID-19 has been a big headache for human beings because of its increasing impacts on public health, economy and education. Many scientists from different sectors are performing research to resolve this problem by discovering its medicine or other means to get rid of this pandemic. The most effective measures to control the spreading of $\mathrm{nCoV}$ are (a) to obtain its vaccination or medication and (b) to break the chain of its transmission by implementing the rules of social distancing and isolation, hand washing, using face mask or PPE and improving body immunity against this disease. As of June 25, 2020, there is no specific antiviral treatment and preventive vaccines. There are many published or ongoing research papers regarding the origin, structure, reproductivity, transmissivity, fatality and socio-economic impacts of SARS-CoV-2 all over the world. Different models of viral propagation and control mechanism based on clinical data analysis and structures of $\mathrm{nCoV}$ components, receptors of hosting cell or enzymic action and possible drugs were investigated or are under study by using the principles of biophysics. A sigmoid logistic model estimates the total cases and total deaths due to nCoV infections in a country with a particular population as averaged from the countries having the cases spiking up and also from the countries having the daily cases slowing down after reaching a peak. The computational study of drug designing and its interactions with $\mathrm{nCoV}$ components are equally important to select the possible drugs before their clinical testing in the COVID-19 patients. The organization of viral genome constituting the mRNA sequence, i.e. the reading frame UTR (5')-S-E-M-N-UTR (3') is a region of interest to understand the viral replication and transcription. The spike protein and main protease of SARS-CoV-2 effectively interacts with the drugs like such as inhibitor N3, remdecivir and favipiravir which are still under pre-clinical or clinical tests against COVID-19 infections. It is important to declare the efficacy and safety of the drugs or vaccines before prescribing for any general public. Due to this fact, it may take long duration to investigate or discover the actual medicine to fight against COVID-19. 


\section{ACKNOWLEDGMENTS}

We gratefully acknowledge Prof. Dr. Binil Aryal, Head, Central Department of Physics, Tribhuvan University for motivation and support to complete this work. Part of the work included here was performed on the supercomputer machine (CDP, TU) supported by the Alexander von Humboldt Foundation, Germany under the equipment grants program and HERP-TU, Nepal.

\section{CONFLICT OF INTEREST}

The authors declared no conflict of interest.

\section{REFERENCES}

Acharya, A., Poudyal, N., Lamichhane, G., Aryal, B., Bhattarai, B. R., Adhikari, B. \& Parajuli, N. (2020). Internet-based online higher education in Nepal amidst COVID-19. https://doi.org/10.35542/ osf.io/r85bc

Al-Khafaji, K., Al-DuhaidahawiL, D. \& Taskin Tok, T. (2020). Using integrated computational approaches to identify safe and rapid treatment for SARS-CoV-2. Journal of Biomolecular Structure and Dynamics, (just-accepted), 1-11.

Asim, M., Sathian, B., Van Teijlingen, E., Mekkodathil, A., Subramanya, S. H. \& Simkhada, P. (2020). COVID-19 pandemic: Public health implications in Nepal. Nepal Journal of Epidemiology, 10(1): 817.

Cai, Q., Yang, M., Liu, D., Chen, J., Shu, D., Xia, J. \& Shen, C. (2020). Experimental treatment with favipiravir for COVID-19: an open-label control study. Engineering. https://doi.org/10.1016/j. eng.2020.03.007

Cascella, M., Rajnik, M., Cuomo, A., Dulebohn, S. C. \& Di Napoli, R. (2020). Features, evaluation and treatment coronavirus (COVID-19). In Statpearls [internet]. StatPearls Publishing.

Chan, J. F. W., Yuan, S., Kok, K. H., To, K. K. W., Chu, H., Yang, J. \& Tsoi, H. W. (2020). A familial cluster of pneumonia associated with the 2019 novel coronavirus indicating person-to-person transmission: a study of a family cluster. The Lancet, 395(10223): 514-523.

Cohen, J. P., Morrison, P. \& Duo, L. (2003). COVID-19 Image Data Collection. arXiv:2003.11597v1. https://arxiv.org/abs/2003.11597. 
Dong, L., Hu, S., \& Gao, J. (2020). Discovering drugs to treat coronavirus disease 2019 (COVID-19). Drug discoveries \& therapeutics, 14(1): 58-60.

Fehr, A. R., \& Perlman, S. (2015). Coronaviruses: an overview of their replication and pathogenesis. In Coronaviruses (pp. 1-23). Humana Press, New York, NY.

Heesterbeek, H., Anderson, R. M., Andreasen, V., Bansal, S., De Angelis, D., Dye, C., Eames, K. T. D., Edmunds, W. J., Frost, S. D. W., Funk, S., Hollingsworth, T. D., House, T., Isham, V., Klepac, P., Lessler, J., Lloyd-Smith, J. O., Metcalf, C. J. E., Mollison, D., Pellis, L., Pulliam, J. R. C., Roberts, M. G., Viboud, C., Isaac New. Ins. IDD Coll. (2015). Modeling infectious disease dynamics in the complex landscape of global health. Science, 347(6227): 4339.

Jin, X., Lian, J. S., Hu, J. H., Gao, J., Zheng, L., Zhang, Y. M., ... \& Yu, G. D. (2020). Epidemiological, clinical and virological characteristics of 74 cases of coronavirus-infected disease 2019 (COVID-19) with gastrointestinal symptoms. Gut, 69(6): 1002-1009.

Jin, Z., Du, X., Xu, Y., Deng, Y., Liu, M., Zhao, Y. \& Duan, Y. (2020). Structure of $\mathrm{M}^{\text {pro }}$ from SARS-CoV-2 and discovery of its inhibitors. Nature. 1-5.

Khadka, S., Hashmi, F.K. \& Usman, M. (2020). Preventing COVID-19 in low- and middle-income countries. Drugs Their Perspect, 36: 250-252.

Koirala, J. \& Acharya, S. (2020). Impact of Novel Corona Virus (COVID-19 or 2019-nCoV) on Nepalese economy. Available at SSRN 3560638.

Koirala, J., Acharya, S., Neupane, M., Phuyal, M., Rijal, N. \& Khanal, U. (2020). Government preparedness and response for 2020 pandemic disaster in Nepal: a case study of COVID-19. Available at SSRN 3564214.

Lamichhane, Tika Ram; \& Ghimire, Madhav Prasad (2020): Structural analysis of COVID-19 main protease and its interaction with the inhibitor N3. ChemR, xiv. https://doi.org/10.26434/ chemrxiv.12400604.v1

Ledford, H. (2020). Coronavirus breakthrough: dexamethasone is first drug shown to save lives. Nature, 582: 469. https://www.nature. com/articles/d41586-020-01824-5 
Li, Q., Guan, X., Wu, P., Wang, X., Zhou, L., Tong, Y. \& Xing, X. (2020). Early transmission dynamics in Wuhan, China, of novel coronavirus-infected pneumonia. New England Journal of Medicine. https://doi.org/10.1056/NEJMoa2001316

Liu, Y., Gayle, A. A., Wilder-Smith, A. \& Rocklöv, J. (2020). The reproductive number of COVID-19 is higher compared to SARS coronavirus. Journal of Travel Medicine, 27(2).

Ministry of Health and Population, Nepal (2020). https://covid19.mohp. gov.np/\#/

Mirza, M. U. \& Froeyen, M. (2020). Structural elucidation of SARSCoV-2 vital proteins: Computational methods reveal potential drug candidates against main protease, Nsp12 polymerase and Nsp13 helicase. Journal of Pharmaceutical Analysis. https://doi. org/10.1016/j.jpha.2020.04.008

Mousavizadeh, L. \& Ghasemi, S. (2020). Genotype and phenotype of COVID-19: Their roles in pathogenesis. Journal of Microbiology, Immunology and Infection. https://doi.org/10.1016/j.jmii. 2020.03. 022

Muralidharan, N., Sakthivel, R., Velmurugan, D. \& Gromiha, M. M. (2020). Computational studies of drug repurposing and synergism of lopinavir, oseltamivir and ritonavir binding with SARS-CoV-2 Protease against COVID-19. Journal of Biomolecular Structure and Dynamics. 1-6.

Neupane, B. B., Mainali, S., Sharma, A. \& Giri, B. (2019). Optical microscopic study of surface morphology and filtering efficiency of face masks. PeerJ, 7, e7142.

Neupane, B. \& Giri, B. (2020). Current understanding on the effectiveness of face masks and respirators to prevent the spread of respiratory viruses.https://doi.org/10.31224/osf.io/h3wgc

Peng, L., Yang, W., Zhang, D., Zhuge, C.\&Hong, L. (2020). Epidemic analysis of COVID-19 in China by dynamical modeling. arXiv:2002.06563. https://arxiv.org/abs/2002.06563v1

Pun, S. B., Mandal, S., Bhandari, L., Jha, S., Rajbhandari, S., Mishra, A. K. \& Shah, R. (2020). Understanding COVID-19 in Nepal. Journal of Nepal Health Research Council, 18(1): 126-127. 
Shang, J., Ye, G., Shi, K., Wan, Y., Luo, C., Aihara, H. \& Li, F. (2020). Structural basis of receptor recognition by SARSCoV-2. Nature, 581(7807): 221-224.

Shrestha, R., Shrestha, S., Khanal, P., \& KC, B. (2020). Nepal's First Case of COVID-19 and public health response. Journal of Travel Medicine, 27(3): 24.

Somsen, G. A., Rijn, C. van, Kooij, S., Bem, R. A. \& Bonn, D. (2020). Small droplet aerosols in poorly ventilated spaces and SARSCoV-2 transmission, Lancet Respir. Med. https://doi.org/10.1016/ S2213-2600(20)30245-9

Vespignani, A., Tian, H., Dye, C., Lloyd-Smith, J. O., Eggo, R. M., Shrestha, M., Scarpino, S. V., Gutierrez, B., Kraemer, M. U. G., Wu, J., Leung, K.\& Leung, G. M. (2020). Modelling COVID-19. Nat. Rev. Phys, 2: 280-281.

Walls, A. C., Park, Y. J., Tortorici, M. A., Wall, A., McGuire, A. T. \&Veesler, D. (2020). Structure, function, and antigenicity of the SARS-CoV-2 spike glycoprotein. Cell. https://doi.org/10.1016/j.cell.2020.02.058

Wang, Y., Zhang, D., Du, G., Du, R., Zhao, J., Jin, Y. \& Hu, Y. (2020). Remdesivir in adults with severe COVID-19: a randomised, doubleblind, placebo-controlled, multicentre trial. Lancet (London, England), 395(10236): 1569-1578.

World Health Organization (2020). https:/www.who.int/emergencies/ diseases/novel-coronavirus-2019, (Accessed: 23.06.2020)

Worldometers (2020). https://www.worldometers.info/coronavirus/ country/nepal. (Accessed: 23.06.2020).

Wu, C., Liu, Y., Yang, Y., Zhang, P., Zhong, W., Wang, Y., ... \& Zheng, M. (2020). Analysis of therapeutic targets for SARSCoV-2 and discovery of potential drugs by computational methods. Acta Pharmaceutica Sinica B. https://doi.org/10.1016/j. apsb.2020.02.008

Yan, R., Zhang, Y., Li, Y., Xia, L., Guo, Y., \& Zhou, Q. (2020). Structural basis for the recognition of SARS-CoV-2 by full-length human ACE2. Science, 367(6485):1444-1448. 